\title{
Time to treatment of uveal melanoma in the United Kingdom
}

\section{Abstract}

Purpose To measure delay before treatment of uveal melanoma.

Methods A retrospective study was carried out of patients referred for diagnosis and treatment of uveal melanoma at the Liverpool Ocular Oncology Centre (LOOC), a tertiary adult ocular oncology service. Participants were patients with uveal melanoma presenting to an optometrist or general practitioner in the United Kingdom between 1997 and 2000. Main outcome measures were delay at each stage of the referral process according to route of referral and tumour size. Results Delays greater than 2 weeks occurred (1) between presentation to the optometrist or general practitioner and the ophthalmologist's examination in $50 \%$ of patients; (2) between the ophthalmologist 's examination and receipt of the referral at the ocular oncology centre in $34 \%$; (3) between receipt of referral at the oncology centre and first assessment at that centre in 16\%; and (4) between first assessment at the oncology centre and treatment in $8 \%$. The waiting time for the ophthalmologist's examination was greater if the optometrist referred the patient via the general practitioner instead of directly. The median overall delays between presentation and treatment were 129 days for small tumours, $\mathbf{5 0}$ days for medium-sized tumours and 34 days for large tumours.

Conclusions Many patients with uveal melanoma experience long delays before treatment. Patients wait longer to see an ophthalmologist if their tumour is small or if they are referred by the optometrist to the general practitioner instead of directly to the ophthalmologist. Several patients also experience long delays after seeing the ophthalmologist, some having a large tumour by the time of teatment.

Key words Adult, Analysis, Diagnosis, Eye neoplasms, Human, Melanoma, Patients, Therapy, Time

Uveal melanomas are diagnosed in approximately 6 people per million per year. ${ }^{1}$ About $80 \%$ occur in the choroid, $12 \%$ in the ciliary body and $8 \%$ in the iris. Most patients present with visual symptoms due to the effects of the tumour on the retina or lens, but in advanced stages there can be pain as a result of glaucoma or inflammation. Previously, the standard form of treatment was enucleation. Today, there is a preference for 'conservative' treatment, which is aimed at conserving the eye with as much useful vision as possible. ${ }^{2}$ The range of conservative treatments includes plaque or proton beam radiotherapy, transscleral or trans-retinal local resection, and various forms of phototherapy, such as transpupillary thermotherapy. The prospects for preserving the eye diminish greatly once the tumour is more than $15 \mathrm{~mm}$ in diameter or more than $8 \mathrm{~mm}$ in thickness. Tumours exceeding these dimensions are also associated with a 5-year mortality greater than $50 \% .^{3}$ For these reasons, early diagnosis and treatment are as vitally important as with other forms of cancer.

In the United Kingdom, patients with uveal melanoma do not present directly to the ophthalmologist but initially visit their general practitioner or optometrist. Most general practitioners would refer the patient directly to an ophthalmologist, although occasionally referral is to an optometrist. Until recently, optometrists were obliged to refer patients to the general practitioner for onward referral to the ophthalmologist, although there is now an increasing tendency for patients to be referred directly to the ophthalmologist. Previously, patients with uveal melanoma were managed by the general ophthalmologist at the patient's local hospital. Today, however, most patients are referred to an ocular oncology centre for specialised care. Treatment is usually performed at the oncology centre, unless enucleation is required or if the patient or the referring ophthalmologist has expressed a preference for the surgery to be performed at the patient's local hospital.

In view of recent government guidelines regarding expedited management of patients with cancer, this study was performed to determine the overall delay before treatment and also the time intervals (1) between presentation to a general practitioner or optometrist and the ophthalmologist's examination; (2) between first
Bertil Damato, PhD,

FRCOphth

Liverpool Ocular Oncology Centre

Royal Liverpool University Hospital

Prescot Street Liverpool L7 8XP, UK

e-mail: Bertil.Damato@ btinternet.com

Received: 5 July 2000 Accepted in revised form: 19 September 2000 
Table 1. Time in days between presentation and treatment according to tumour size at first assessment at the ocular oncology centre

\begin{tabular}{|c|c|c|c|c|c|c|c|c|c|c|}
\hline & \multicolumn{2}{|c|}{$\begin{array}{l}\text { From presentation } \\
\text { to GP or optometrist } \\
\text { to tumour treatment }\end{array}$} & \multicolumn{2}{|c|}{$\begin{array}{c}\text { From presentation } \\
\text { to GP or optometrist } \\
\text { to ophthalmologist's } \\
\text { exam. }\end{array}$} & \multicolumn{2}{|c|}{$\begin{array}{l}\text { From ophthalmologist's } \\
\text { exam. to receipt of } \\
\text { referral at oncology } \\
\text { centre } \\
\end{array}$} & \multicolumn{2}{|c|}{$\begin{array}{l}\text { From receipt of } \\
\text { referral at oncology } \\
\text { centre to ocular } \\
\text { oncologist's exam. }\end{array}$} & \multicolumn{2}{|c|}{$\begin{array}{l}\text { From ocular } \\
\text { oncologist's exam. at } \\
\text { oncology centre to } \\
\text { treatment of tumour }\end{array}$} \\
\hline & $\begin{array}{l}\text { Median } \\
\text { (inter- } \\
\text { quartile } \\
\text { range) }\end{array}$ & $\begin{array}{c}\text { No. } \\
\text { treated in } \\
2 \text { weeks } \\
(\%)\end{array}$ & $\begin{array}{l}\text { Median } \\
\text { (inter- } \\
\text { quartile } \\
\text { range) }\end{array}$ & $\begin{array}{c}\text { No. } \\
\text { seen in } \\
2 \text { weeks } \\
(\%)\end{array}$ & $\begin{array}{l}\text { Median } \\
\text { (inter- } \\
\text { quartile } \\
\text { range) }\end{array}$ & $\begin{array}{l}\text { No. } \\
\text { received in } \\
2 \text { weeks } \\
(\%)\end{array}$ & $\begin{array}{l}\text { Median } \\
\text { (inter- } \\
\text { quartile } \\
\text { range) }\end{array}$ & $\begin{array}{c}\text { No. } \\
\text { seen in } \\
2 \text { weeks } \\
(\%)\end{array}$ & $\begin{array}{l}\text { Median } \\
\text { (inter- } \\
\text { quartile } \\
\text { range) }\end{array}$ & $\begin{array}{c}\text { No. } \\
\text { treated in } \\
2 \text { weeks } \\
(\%)\end{array}$ \\
\hline $\begin{array}{l}\text { Small } \\
(n=60)\end{array}$ & $129(45-452)$ & $3(5)$ & $32(13-129)$ & $18(30)$ & $13(5-197)$ & $32(53)$ & $11(6-17)$ & $46(77)$ & $1(1-1)$ & 58 (97) \\
\hline $\begin{array}{l}\text { Medium } \\
(n=171)\end{array}$ & $50(24-133)$ & $17(10)$ & $14(1-62)$ & $88(52)$ & $8(4-22)$ & $113(66)$ & $10(6-13)$ & $145(85)$ & $1(1-2)$ & $156(91)$ \\
\hline $\begin{array}{l}\text { Large } \\
(n=73)\end{array}$ & $34(17-135)$ & $10(14)$ & $3(0-51)$ & $47(64)$ & $6(2-18)$ & $54(74)$ & $9(6-13)$ & $63(86)$ & $1(1-4)$ & 65 (89) \\
\hline $\begin{array}{l}\text { Total } \\
(n=304)\end{array}$ & $53(23-152)$ & $30(10)$ & $14(1-61)$ & $153(50)$ & $8(3-26)$ & $199(66)$ & $10(6-13)$ & $254(84)$ & $1(1-2)$ & $279(92)$ \\
\hline
\end{tabular}

ophthalmologist's examination and receipt of referral at the ocular oncology centre; (3) between receipt of referral at the ocular oncology centre and assessment by the ocular oncologist at that centre; and (4) between assessment by the ocular oncologist and definitive tumour treatment.

\section{Methods}

New patients attending the Liverpool Ocular Oncology Centre (LOOC) between June 1997 and April 2000 were asked about the nature and duration of any symptoms, the date of their presentation to a general practitioner or optometrist, the outcome of the initial consultation, and the sequence of events leading to their referral to the oncology centre. If they were unable to remember the precise date when an event occurred, they were asked to give an approximate indication. The date of the general practitioner's involvement in the referral process was taken either as the date of the patient's consultation or, if such a visit did not take place, the date when the optometrist telephoned the general practitioner about the patient's suspected diagnosis. The structured interview was conducted during the patient's first visit to the ocular oncology centre by a specialist ophthalmic registrar or specialist ocular oncology nurse, using a proforma. The recorded information was checked minutes later by the ocular oncologist (B.D.) in the presence of the patient and subsequently computerised in a customised ocular oncology database by a full-time data manager. Information on visual acuity, tumour dimensions and other characteristics was computerised by the ocular oncologist at the same clinic visit.

Measurements of tumour dimensions were obtained by ultrasonography performed by the ocular oncologist at the patient's first visit to the ocular oncology centre. In keeping with conventional protocol, ${ }^{3}$ tumours were categorised as: 'small' if they were less than $10.5 \mathrm{~mm}$ wide in their largest basal diameter and less than $2.5 \mathrm{~mm}$ thick; 'medium' if the basal diameter was between $10.5 \mathrm{~mm}$ and $15.4 \mathrm{~mm}$ or if the thickness was between 2.5 and $8.4 \mathrm{~mm}$; and 'large' if the basal diameter or thickness, or both, exceeded these figures. Patients were included in the study if they presented to an optometrist or general practitioner and if they were diagnosed clinically by the ocular oncologist as suffering from uveal melanoma. They were excluded if their tumour was initially detected by an ophthalmologist while they were being treated for an unrelated condition, if they did not reside in the United Kingdom, if their symptoms started before 1990, if they were treated previously, either at the LOOC or elsewhere, or if it was not possible to measure tumour dimensions ultrasonographically (in 5 patients).

Statistical analysis was performed with SPSS for Windows (version 10.0.5). The chi-squared test was used to compare differences in the numbers of patients progressing from one stage in the referral process to the next within a 2 week period. A correction for continuity was included in the chi-squared test for all $2 \times 2$ tables. The Mann-Whitney test was used to compare time intervals. Tests were two-sided, with $5 \%$ significance level.

\section{Results}

The sample numbered 304 patients (157 female, 147 male), residing in England (80\%), Scotland ( $9 \%)$, Wales $(5 \%)$ and Northern Ireland $(6 \%)$. The tumours were located in the right eye in 168 patients and in the left eye in 136. At the ocular oncologist's examination soon before treatment of the tumour, the mean age was 59.7 years (SD 13.9; range 22-94). The average largest basal tumour diameter and average thickness were $11.6 \mathrm{~mm}$ and $4.9 \mathrm{~mm}$ respectively. The patients were treated by plaque radiotherapy $(41 \%)$, enucleation $(31 \%)$, proton beam radiotherapy $(16 \%)$, transscleral local resection $(8 \%)$, endoresection $(3 \%)$ and trans-pupillary thermotherapy $(1 \%)$.

The overall time between presentation with symptoms to a general practitioner or optometrist or detection of an asymptomatic tumour by such a practitioner and treatment of the tumour had a median of 53 days (Table 1). This interval exceeded 20 weeks in $45 \%$ 


\begin{tabular}{|c|c|c|c|}
\hline Time interval (days) & $\begin{array}{c}\text { Median } \\
\text { (interquartile range) }\end{array}$ & $\begin{array}{c}\text { No. seen in } \\
2 \text { weeks } \\
(\%)\end{array}$ & $\begin{array}{l}\text { No. seen after } \\
20 \text { weeks } \\
(\%)\end{array}$ \\
\hline \multicolumn{4}{|l|}{ Patients presenting to optometrist } \\
\hline From optometrist to ophthalmologist $(n=117)$ & $7(6-13)$ & $70(60)$ & $10(9)$ \\
\hline From optometrist to general practitioner $(n=97)^{a}$ & $5(1-28)$ & $69(71)$ & $8(8)$ \\
\hline From general practitioner to ophthalmologist $(n=97)^{a}$ & $23(3-50)$ & $41(42)$ & $8(8)$ \\
\hline \multicolumn{4}{|l|}{ Patients presenting to general practitioner } \\
\hline From general practitioner to ophthalmologist directly $(n=65)$ & $6(1-36)$ & $43(66)$ & $7(11)$ \\
\hline From general practitioner to ophthalmologist via optometrist $(n=20)$ & $18(8-111)$ & $9(45)$ & $2(10)$ \\
\hline
\end{tabular}

${ }^{a}$ Five patients excluded because of missing date of receipt of letter by general practitioner from optometrist.

of patients with small tumours as compared with $23 \%$ of patients with medium-sized or large tumours $\left(\chi^{2}=11.22\right.$, d.f. $=1, p=0.001)$.

The greatest delay occurred between the visit to an optometrist or general practitioner and the ophthalmologist's examination, with $50 \%$ of patients being seen within 2 weeks and $12 \%$ waiting longer than 20 weeks. Patients presenting to the optometrist waited longer to see the ophthalmologist if they were referred via the general practitioner than directly (median delay 34 days vs 6 days; Mann-Whitney, $p<0.0001)$ and were also less likely to be seen within 2 weeks ( $30 \%$ vs $60 \%$; $\chi^{2}=17.84$, d.f. $\left.=1, p<0.0001\right)$. Patients referred by optometrists via the general practitioner waited a median of 5 days for the general practitioner to become involved in their referral (i.e. in person or by telephone) and then waited a median of 23 days to see the ophthalmologist (Table 2). Patients referred directly from the general practitioner to the ophthalmologist waited longer if they had been referred to the general practitioner by an optometrist than if they presented directly to the general practitioner (median delay 23 days vs 6 days;

Mann-Whitney, $p=0.029$ ). Twenty patients presenting to the general practitioner were referred to an optometrist instead of an ophthalmologist and this increased the median delay from 6 days to 18 days (Mann-Whitney, $p=0.02$ ). Patients with larger tumours were more likely to be seen by an ophthalmologist within 2 weeks than patients with small tumours (large $64 \%$ vs medium $52 \%$ vs small $30 \% ; \chi^{2}=15.775$, d.f. $=2, p<0.0001$ ).

After their first visit to the ophthalmologist, $66 \%$ of patients were referred to the ocular oncology centre within 2 weeks. Delay at this stage was more likely to exceed 20 weeks if the tumour was small than if it was medium-sized or large $\left(27 \%\right.$ vs $7 \% ; \chi^{2}=16.15$, d.f. $=1$, $p<0.0001$ ).

Eighty-four per cent of patients were seen at the ocular oncology centre within 2 weeks of receipt of referral. There were 2 patients with a delay of more than 20 weeks. These 2 patients were elderly and had both delayed the visit themselves. Most patients were treated 1 day after their first assessment at the ocular oncology centre, with $92 \%$ receiving treatment within 2 weeks. Reasons for delayed treatment included: referral for enucleation at the patient's own hospital, at the request of the patient or the referring ophthalmologist (7 patients); patient's request for delay, because of vacation or need to consider therapeutic options (3 patients); need to manufacture customised radioactive plaque (2 patients); observation (2 patients); conjunctivitis (1 patient) and Christmas/New Year vacation (1 patient). Only 9 operations ( $3 \%$ ) were delayed because of insufficient theatre time to deal with a cluster of referrals.

\section{Discussion}

After initial presentation to an optometrist or general practitioner, only $50 \%$ of patients with uveal melanoma were seen by an ophthalmologist within 2 weeks and $27 \%$ waited more than 20 weeks for treatment.

This study includes patients from all over the United Kingdom and is considerably larger and more detailed than two previous studies performed at the same institution. ${ }^{4,5}$ The present study has two weaknesses. First, the sample inevitably includes some patients whose tumour was not detected when they first presented to the optometrist or general practitioner and were referred either because of symptoms arising from unrelated, concurrent disease or because their tumour was misdiagnosed. Second, some tumours categorised as large or medium-sized at the time of treatment may have been in a different category when the patient was managed by the general practitioner, optometrist or ophthalmologist.

Much of the delay occurred before the patient was seen by the ophthalmologist. Patients referred by optometrists via the general practitioner waited considerably longer than those referred directly. There would seem to a case for all patients with suspected melanoma being referred directly to the ophthalmologist by the optometrist, with appropriate notification of the general practitioner by post or telephone. Patients with small tumours waited longer to see the ophthalmologist than those with larger tumours, perhap because small tumours were deemed to be less dangerous. Since it is not known when uveal melanomas metastasise, all patients with suspected melanoma should be treated urgently, irrespective of tumour size. In some patients the long delay may have occurred because the tumour was not detected by the optometrist or general practitioner. In this sample, $52 \%$ of patients presenting to the general practitioner and $21 \%$ of those presenting to the optometrist reported that their tumour had not been 
detected when they presented with symptoms (Damato, unpublished data). The question arises as to whether or not it is acceptable for patients with recent visual symptoms to be referred without proper ophthalmoscopy, that is, performed with mydriasis and examining not only the optic disc and macula but also the peripheral fundus.

Many patients experienced considerable delay after being seen by the referring ophthalmologist. With small tumours, this may be reasonable because documentation of tumour growth by sequential examination is a recognised method of differentiating large, benign naevi from small, malignant melanomas. Many patients with delayed referral, however, were found to have medium-sized or large tumours on arrival at the oncology centre. Although no formal audit was performed, the main reasons for delay seemed to be (1) referral from the eye casualty to the ophthalmic clinic at the same hospital; (2) awaiting results of special investigations, such as fluorescein angiography, ocular ultrasonography and liver scanning; and (3) delays in preparing and posting the referral letter. The referral process would probably be expedited if patients were to be referred immediately, by telephone, this referral being followed by a mailed letter, with all necessary investigations performed at the oncology centre.

The time between receipt of the referral and assessment at the ocular oncology centre exceeded 2 weeks in $16 \%$ of patients. The main reasons for such delays were public holidays occurring on a Monday, which is when the weekly clinic was held, and other occasions when the ocular oncologist was on leave, the service being consultant-driven. Treatment within a short period of assessment was achieved by keeping an all-day operating theatre session for new patients seen on the previous day, with the facility to use a second operating theatre session a few days later. Treatment was occasionally delayed if the patient requested time to consider the various therapeutic option and to listen to a tape-recording of the consultation (which was routinely provided to all new patients).

Many factors influence ocular outcome and survival in patients with uveal melanoma, so that it is not possible to demonstrate the consequences of delayed treatment, except anecdotally. Nevertheless, during their consultation at the oncology centre many patients complained bitterly about long delays in the referral process, spontaneously reporting psychological distress and expressing fears about missed opportunities for conserving vision and preventing metastasis.

Statistical advice was provided by Ian Campbell. Jane Humphreys assisted with the retrieval of information, which was computerised by Gary Cheetham. Funding: The Liverpool Ocular Oncology Centre is partly funded by the National Supraregional Committee Advisory Group (NSCAG). Competing interests: None declared.

\section{References}

1. Scotto J, Fraumeni JF Jr, Lee JAH. Melanomas of the eye and other noncutaneous sites: epidemiologic aspects. J Natl Cancer Inst 1976;56:489-91.

2. Damato BE. Ocular tumours: diagnosis and treatment. Oxford: Butterworth-Heinemann, 2000:74-87.

3. Diener West M, Hawkins BS, Markowitz JA, Schachat AP. A review of mortality from choroidal melanoma. II. A metaanalysis of 5-year mortality rates following enucleation, 1966 through 1988. Arch Ophthalmol 1992;110:245-50.

4. Holden R, Damato BE. Preventable delays in the treatment of intraocular melanoma in the UK. Eye 1996;10:127-9.

5. Ah-Fat FG, Damato BE. Delays in the diagnosis of uveal melanoma and effect on treatment. Eye 1998;12:781-2. 